Pacific Journal of Mathematics

RELATIONS AMONG CERTAIN RANGES OF VECTOR

A. DVOREIZKY, A. WALD AND J. WOLFOWITZ 


\title{
RELATIONS AMONG CERTAIN RANGES OF VECTOR MEASURES
}

\author{
A.DVORETZKY, A. WALD, AND J.WOLFOWITZ
}

1. Introduction and definitions. The purpose of the present paper is to prove certain measure theoretical results concerning ranges of measures. One of our results (the closure and convexity result implied by Theorem 4) may be regarded as a generalization of a theorem of Liapounoff [5]. The results obtained here have applications to statistics and the the ory of games.

Throughout this paper $\{x\}=X$ denotes an arbitrary space, and $\{S\} \equiv \Xi$ denotes a Borel field of subsets of $X$; that is, $\subseteq$ is a nonempty family of subsets of $X$ which is closed with respect to the operations of complementation (with respect to $X$ ) and countable union. The phrase, $S$ is measurable, will be used as synonymous with $S \in \widetilde{S}$.

A real-valued countably additive set function defined for all measurable sets will be called a measure. Thus we admit measures assuming negative or infinite values. A measure cannot, however, assume the value $+\infty$ for one measurable set and $-\infty$ for another such set, since in such a case additivity cannot be defined satisfactorily. A measure is called finite if it assumes finite values for all measurable sets. It is called nonnegative if it assumes nonnegative values for all such sets.

We say that $f(x)$ is a measurable function if it is real-valued, defined for all $x \in X$, and if, moreover, the set $f_{c}$ of all $x \in X$ for which $f(x)<c$ is measurable for every real number $c$. A step function is a measurable function which assumes only a finite number of values.

If $n$ is a positive integer and $\eta_{j}(x)(j=1, \cdots, n)$ are nonnegative measurable functions satisfying

$$
\eta_{1}(x)+\cdots+\eta_{n}(x)=1 \quad \text { for every } x \in X,
$$

then $\eta(x)=\left[\eta_{1}(x), \cdots, \eta_{n}(x)\right]$ will be called a probability $n$-vector. The functions $\eta_{j}(x)$ are called the components of this vector. If all the components of

Received July 20, 1950. This research was sponsored (in part) by the Office of Naval Research; the main results of the paper were announced without proof in an earlier publication [1]. The first author is on leave of absence from the Hebrew University, Jerusalem, Israel.

Pacific J. Math. 1 (1951), 59-74. 
$\eta(x)$ are step functions, then $\eta(x)$ is called a (probability) step $n$-vector. We shall occasionally denote such vectors by $\eta^{0}(x)$. If, in particular, all components of $\eta(x)$ assume only the two values zero and one, that is, if for every $x \in X$ one $\eta_{j}(x)$ is equal to one and all others vanish, then $\eta(x)$ is called a pure n-vector. Such vectors will be denoted by $\eta^{*}(x)$. If the $j$ th component $(j=1, \cdots, n)$ of $\eta^{*}(x)$ is considered as the characteristic function of a set $S_{j}$, then the sets $S_{1}, \cdots, S_{n}$ are measurable and disjoint and their union is $X$. Conversely, if $S_{1}, \cdots, S_{n}$ is a decomposition of $X$ into $n$ disjoint measurable sets, and $\eta_{j}^{*}(x)$ is the characteristic function of $S_{j}$, then $\eta^{*}(x)=\left[\eta_{1}^{*}(x), \cdots, \eta_{n}^{*}(x)\right]$ is a pure $n$-vector. We therefore call $\eta^{*}(x)$ also a decomposition n-vector or, more specifically, a decomposition $n$-vector corresponding to the decomposition $X=S_{1} \cup \cdots \cup S_{n}$.

Let $\mu_{k}(S)(k=1, \cdots, p)$ be a finite set of measures, and let $\eta(x)$ be a probability $n$-vector. We denote by $v(\eta)=v\left(\eta ; \mu_{1}, \cdots, \mu_{p}\right)$ the $n p$ dimensional vector (or point in $n p$ space),

$$
\begin{aligned}
{\left[\int_{X} \eta_{1}(x) d \mu_{1}(x), \cdots,\right.} & \int_{X} \eta_{1}(x) d \mu_{p}(x), \\
& \left.\int_{X} \eta_{2}(x) d \mu_{1}(x), \cdots, \quad \int_{X} \eta_{p}(x) d \mu_{p}(x)\right] .
\end{aligned}
$$

The set of all points $v(\eta)=v\left(\eta ; \mu_{1}, \cdots, \mu_{p}\right)$ corresponding to all probability $n$-vectors $\eta(x)$ is called the $n$-range of $\mu_{1}, \cdots, \mu_{p}$ and is further denoted by $V_{n}\left(\mu_{1}, \cdots, \mu_{p}\right)$ or, more concisely, by $V_{n}$. In the same way we define the step $n$-range of $\mu_{1}, \cdots, \mu_{p}$ as the set of all points $v\left(\eta^{0}\right)=v\left(\eta^{0} ; \mu_{1}, \cdots, \mu_{p}\right)$ corresponding to all step $n$-vectors $n^{0}(x)$, and denote it by $V_{n}^{0}\left(\mu_{1}, \cdots, \mu_{p}\right)$ or $V_{n}^{0}$. Similarly $V_{n}^{*}$ or $V_{n}^{*}\left(\mu_{1}, \cdots, \mu_{p}\right)$ denotes the set of all points

$$
v\left(\eta^{*}\right)=v\left(\eta^{*} ; \mu_{1}, \cdots, \mu_{p}\right)
$$

corresponding to all pure $n$-vectors $\eta^{*}(x)$ and is called the decomposition n-range of $\mu_{1}, \cdots, \mu_{p}$. When no confusion is possible we replace $n$-range in the above terms by range.

It is shown in Section 2 that if $\mu_{1}, \cdots, \mu_{p}$ are finite measures then the range: $V_{n}\left(\mu_{1}, \cdots, \mu_{p}\right)$ is compact and convex and coincides with the step-range $V_{n}^{0}\left(\mu_{1}, \cdots, \mu_{p}\right)$. Actually a stronger result is proved; this states that the points $v\left(\eta ; \mu_{1}, \cdots, \mu_{p}\right)$ for which the components of $\eta(x)$ assume at most $2^{n p-p+1}$ 
different values already fill $V_{n}$. Applying a theorem of Liapounoff we deduce, in Section 3 , the result that if the measures are atomless then the decomposition range $V_{n}^{*}\left(\mu_{1}, \cdots, \mu_{p}\right)$ is identical with $V_{n}\left(\mu_{1}, \cdots, \mu_{p}\right)$. This result is extended in Section 4 to arbitrary (not necessarily finite) atomless measures. Applications of these results to statistics and the theory of games are briefly indicated in Section 5.

2. Identity of the step range and the range for finite measures. First, we prove the following result.

THEOREM 1. If $\mu_{1}, \cdots, \mu_{p}$ are finite measures, then for every $n$, the range $V_{n}\left(\mu_{1}, \cdots, \mu_{p}\right)$ is a compact and convex set in Euclidean np dimensional space.*

Proof. Let $A=v(\eta)$ and $A^{\prime}=v\left(\eta^{\prime}\right)$ be any two points of $V_{n}$. Then every point of the segment joining them is represented vectorially by $c A+(1-c) A^{\prime}$, with $0<c<1$. But such a point is clearly $v\left[c \eta+(1-c) \eta^{\prime}\right]$ and, since $c \eta+(1-c) \eta^{\prime}$ is a probability $n$-vector, the point also belongs to the range. Thus $V_{n}$ is convex.

The proof of compactness is more difficult. We start by establishing a lemma on sequences of measures.

LEMMA 1. Let $\{B\}=\Re$ be a Borel field of subsets of $X$ generated by countably many sets. Let $\mu^{t}(t=1,2, \cdots)$ and $\mu$ be measures over $B$ satisfying, for all $B \in \mathfrak{B}$,

$$
0 \leq \mu^{t}(B) \leq \mu(B)<\infty \quad(t=1,2, \cdots)
$$

Then there exists a measure $\nu$ over $B$ satisfying

$$
0 \leq \nu(B) \leq \mu(B) \quad \text { for all } B \in B
$$

and a sequence of integers $t_{q}(q=1,2, \cdots)$ satisfying

$$
0<t_{1}<t_{2}<\cdots<t_{q}<t_{q+1}<\cdots
$$

such that

$$
\lim _{q=\infty} \mu^{t} q(B)=\nu(B) \quad \text { for every } \quad B \in \mathbb{B}
$$

* For the special case when $X$ is a finite-dimensional Euclidean space and all the $\mu_{k}$ are absolutely continuous, this theorem follows from Theorems 3.1 and $3.2 \mathrm{a}$ of [6] 
The proof of the lemma proceeds as follows. Let $B_{0}=X, B_{1}, \cdots, B_{n} \cdots$ be a countable basis of $\Re$. Then, according to the well-known diagonal procedure of Cantor, there exists a sequence (4) for which

$$
\beta_{r}=\lim _{q=\infty} \mu^{t_{q}}\left(B_{r}\right)
$$

exists for $r=0,1,2, \cdots$. To prove the existence of the limit in (5) for every $B$, and the fact that this limit is a measure, it suffices to show that: (a) if $\mu^{t} q(B)$ tends to a limit as $q \rightarrow \infty$, then so does $\mu^{t} q(\bar{B})$ where $\bar{B}$ is the complement of $B$ with respect to $X$; and (b) if $B^{s}(s=1,2, \cdots)$ are disjoint sets of $B$ for which $\lim _{q=\infty} \mu^{t} q\left(B^{s}\right)$ exists for $s=1,2, \cdots$, then we have also

$$
\lim _{q=\infty} \mu^{t_{q}}\left(\bigcup_{s=1}^{\infty} B^{s}\right)=\sum_{s=1}^{\infty} \lim _{q=\infty} \mu^{t_{q}}\left(B^{s}\right)
$$

Now (a) follows immediately when we write $\mu^{t}(\bar{B})=\mu^{t}(X)-\mu^{t}(B)$ and observe that $\mu^{t} q(X)$ has the limit $\beta_{0}$. To prove (6) it is sufficient to observe that the functions $\mu^{t}$ are countably additive, that by (2) we have $\mu^{t} q\left(B^{s}\right) \leq \mu\left(B^{s}\right)$, and that $\sum_{s=1}^{\infty} \mu\left(B^{s}\right)$ is a convergent series of nonnegative terms. (This is the standard bounded convergence argument.) Since (2) and (5) obviously imply (3), the proof of Lemma 1 is completed.

Let now $\eta^{t}(x)(t=1,2, \cdots)$ be any sequence of probability $n$-vectors. The compactness of $V_{n}$ will be proved if we show that there exist a probability $n$-vector and a sequence (4) satisfying

$$
\lim _{q=\infty} \int_{X} \eta_{J}^{t_{q}}(x) d \mu_{k}(x)=\int_{X} \eta_{j}(x) d \mu_{k}(x) \quad(j=1, \cdots, n ; k=1, \cdots, p) .
$$

Denote by $B_{j, \rho}^{t}(t=1,2, \cdots ; j=1, \cdots, n ; \rho$ rational with $0 \leq \rho \leq 1)$ the set of all $x$ for which $\eta_{j}^{t}(x) \leq \rho$, and let $\{B\}=\mathscr{B} \subset \subseteq$ be the smallest Borel field containing these sets. Write $\left|\mu_{k}\right|$ for the absolute measure* associated with $\mu_{k}$. Put $\mu(B)=\left|\mu_{1}\right|(B)+\cdots+\left|\mu_{p}\right|(B)$ for every $B \in \mathscr{B}$. Then $B, \mu$, and

* That is, $\left|\mu_{k}\right|(S)=\sup \left[\left|\mu_{k}\left(S^{\prime}\right)\right|+\left|\mu_{k}\left(S^{\prime \prime}\right)\right|\right]$ for all decompositions of $S$ into two disjoint measurable sets $S^{\prime}$ and $S^{\prime \prime}$. 
$\mu^{t}$, defined by

$$
\mu^{t}(B)=\int_{B} \eta_{1}^{t}(x) d \mu(x) \quad(t=1,2, \cdots, B \in \mathbb{B})
$$

satisfy the conditions of Lemma 1 . Hence there exists a nonnegative measure $\nu_{1}$ over $B$ and a sequence (4), for which

$$
\lim _{q=\infty} \int_{B} \eta_{1}^{t_{q}}(x) d \mu(x)=\nu_{1}(B)
$$

for every $B \in \mathfrak{B}$.

Again applying Lemma 1 , we can extract from the sequence $t_{q}$ a further subsequence for which (8) holds with the subscript 1 replaced by 2 . Repeating this $n-1$ times, and again denoting, for simplicity of writing, the final subsequence by $t_{q}$, we see that there exist nonnegative measures $\nu_{1}, \cdots, \nu_{n}$ over $B$ and a sequence (4) satisfying

$$
\lim _{y=\infty} \int_{B} \eta_{j}^{t_{q}}(x) d \mu(x)=\nu_{j}(B)
$$

$$
(j=1, \cdots, n)
$$

for every $B \in B$. Clearly, we have

$$
\nu_{1}(B)+\cdots+\nu_{n}(B)=\mu(B)
$$

By the Radon-Nikodym theorem there exist $\mathfrak{B}$-measurable functions $f_{j}(x)$ $(j=1, \cdots, n)$ such that

$$
\nu_{j}(B)=\int_{B} f_{j}(x) d \mu(x) \quad(j=1, \cdots, n)
$$

for every $B \in \Re$. Since the $\nu_{j}$ are nonnegative measures, we may assume that the $f_{j}$ are nonnegative functions; and, because of (10), we may further assume that $f_{1}(x)+\cdots+f_{n}(x)=1$ for every $x$. The $f_{j}$ are $\mathfrak{B}$-measurable and are, a fortiori, $\Xi$-measurable; hence $\left[f_{1}(x), \cdots, f_{n}(x)\right]$ is a probability $n$-vector. We denote this vector by $\eta(x)$ and proceed to show that (7) holds with this $\eta$ and the above constructed sequence (4) satisfying (9).

Let $g_{k}(x)(k=1, \cdots, p)$ denote a $\mathbb{B}$-measurable Radon-Nikodym derivative 
$d \mu_{k}(x) / d \mu(x)$. Then, replacing $f_{j}$ in (11) by $\eta_{j}$, we have

$$
\begin{aligned}
\int_{X} \eta_{j}(x) d \mu_{k}(x) & =\int_{X} \eta_{j}(x) g_{k}(x) d \mu(x) \\
& =\int_{X} g_{k}(x) d \int \eta_{j}(x) d \mu(x)=\int_{X} g_{k}(x) d \nu_{j}(x) .
\end{aligned}
$$

Similarly, the left side of (7) may be rewritten as

$$
\lim _{q=\infty} \int_{X} g_{k}(x) d \int \eta_{J}^{t_{q}}(x) d \mu(x)
$$

and thus (7) follows from (9). This completes the proof of Theorem 1.

For any compact convex set $C$ in a Euclidean space, we designate as extreme points of $C$, all those points of $C$ which are not interior points of any segment lying in $C$. Our next result is the following.

THEOREM 2. If the measures $\mu_{1}, \cdots, \mu_{p}$ are finite, and $v(\eta)$ is an extreme point of $V_{n}$, then the set of $x$ for which $0<\eta_{j}(x)<1$ for at least one $j(j=1, \cdots$, $n)$ is a null-set ${ }^{*}$ for each of the measures $\mu_{1}, \cdots, \mu_{p}$. In particular, all extreme points of $V_{n}$ belong to the decomposition range $V_{n}^{*}$.

Proof. Let $Y$ denote the set of $x$ defined in the theorem. If $Y$ is not a null-set for $\mu_{k_{0}}$ with $1 \leq k_{0} \leq \mathrm{p}$, then there exist integers $j_{0}, j_{1}$ with $1 \leq j_{0}<j_{1} \leq n$, a number $\delta>0$, and a measurable set $Z \subset Y$, such that

$$
\delta<\eta_{j}(x)<1-\delta \quad \text { for } x \in Z \quad \text { and } \quad j=j_{0}, j_{1},
$$

and

$$
\mu_{k_{0}}(Z) \neq 0
$$

Let $\zeta=\zeta(x)=\left[\zeta_{1}(x), \cdots, \zeta_{n}(x)\right]$ be the vector defined as follows:

$$
\zeta_{j_{0}}(x)=-\zeta_{j_{1}}(x)=\left\{\begin{array}{l}
\delta \text { if } x \in Z \\
0 \text { if } x \notin Z
\end{array}\right.
$$

\footnotetext{
*A measurable $S$ is a null-set for the measure $\mu$ if $\mu\left(S^{\prime}\right)=0$ for every measurable $S^{\prime} \subset S$.
} 
and all other components vanish identically.

Because of (12), $\eta(x)+\theta \zeta(x)$ is a probability $n$-vector whenever $-1 \leq \theta \leq 1$. Since

$$
\int_{X}\left[\eta_{j_{0}}(x)+\zeta_{j_{0}}(x)\right] d \mu_{k_{0}}(x)-\int_{X}\left[\eta_{J_{0}}(x)-\zeta_{J_{0}}(x)\right] d \mu_{k_{0}}(x)
$$

$$
=2 \delta \mu_{k_{0}}(Z) \neq 0
$$

the points $v(\eta+\zeta)$ and $v(\eta-\zeta)$ are different. But clearly as $\theta$ increases from -1 to +1 the point $v(\eta+\theta \zeta)$ moves from $v(\eta-\zeta)$ to $v(\eta+\zeta)$ along the segment connecting them. Moreover, $v(\eta)$ is the middle point of this segment and thus it cannot be an extreme point of $V_{n}$.

If $v(\eta)$ is an extreme point, then $Y$ is a null-set for all $\mu_{k}$. Therefore, if we put $\eta^{*}(x)=\eta(x)$ for $x \notin Y$ and, say, $\eta_{1}^{*}(x)=1$ for $x \in Y$, the decomposition vector $\eta^{*}(x)$ thus defined satisfies $v\left(\eta^{*}\right)=v(\eta)$. This proves the last assertion of Theorem 2.

ТНЕОRЕМ 3. If the measures $\mu_{1}, \cdots, \mu_{p}$ are finite, then the step-range $V_{n}^{0}$ coincides with the range $V_{n}$. More precisely, every point of $V_{n}$ may be represented as $v\left(\eta^{0}\right)$, where $\eta^{0}$ is a step n-vector whose components assume not more than $2^{n p-p+1}$ different values.

Proof. According to Theorem $1, V_{n}$ is a compact convex set in Euclidean $n p$-dimensional space. However, because of the $p$ equations

$$
\sum_{J=1}^{n} \int_{X} \eta_{J}(x) d \mu_{k}(x)=\mu_{k}(x) \quad(k=1, \cdots, p)
$$

$V_{n}$ lies in an $N=n p-p$ dimensional linear subspace. Hence, according to wellknown facts on convex bodies, every point $P$ of $V_{n}$ may be represented vectorially by

$$
P=c_{1} P_{1}+\cdots+c_{N} P_{N}+c_{N+1} P_{N+1},
$$

where $P_{1}, \cdots, P_{N+1}$ are extreme points of $V_{n}$ and $c_{1}, \cdots, c_{N+1}$ are nonnegative constants whose sum is 1 . According to Theorem 2, we have $P_{r}=v\left(\eta^{*} r\right)$ with $\eta^{*} r$ a decomposition $n$-vector $(r=1,2, \cdots, N+1)$. 
Hence, putting $\eta^{0}=\sum_{r=1}^{N+1} c_{r} \eta^{* r}$, we have $P=v\left(\eta^{0}\right)$. Clearly, for every $x$, every component of $\eta^{0}(x)$ equals $\Sigma_{r \in K} c_{r}$, where $K$ is a subset of $\{1,2, \cdots, N+1\}$. There being $2^{N+1}$ such subsets, Theorem 3 is proved.

3. Identity of the range and the decomposition range for finite atomless measures. A measurable set $S$ is called an atom of the measure $\mu$ if $\mu(S) \neq 0$ and if, moreover, for every measurable $S^{\prime} \subset S$ we have either $\mu\left(S^{\prime}\right)=0$ or $\mu\left(S^{\prime}\right)=\mu(S)$. If the measure $\mu(S)$ has no atoms it is called atomless.

For atomless measures we can improve on The orem 3 by establishing the following result.

THEOREM 4. If $\mu_{1}, \cdots, \mu_{p}$ are finite atomless measures then, for every $n$, the range $V_{n}$ and the decomposition range $V_{n}^{*}$ are identical.

According to Theorem 1 , the common range is convex and compact.

Proof. In view of Theorem 3 it suffices to prove that, in the present case, $V_{n}^{*}=V_{n}^{0}$.

For this purpose we shall use the following fact: If $\mu_{1}, \cdots, \mu_{p}$ are finite and atomless, then, given $0 \leq c \leq 1$, there exists a measurable set $S$ for which

$$
\mu_{k}(S)=c \mu_{k}(X) \quad(k=1, \cdots, p) .
$$

The existence of such a set $S$ follows immediately from a result of Liapounoff [5] (see also [3]) according to which, under the above stated conditions, the set of points $\mu_{1}(S), \cdots, \mu_{p}(S)$ in Euclidean $p$-space corresponding to all measurable $S$ is convex. Indeed, the empty set $\Lambda$ and $X$ are certainly measurable and $(1-c) \mu_{k}(\Lambda)+c \mu_{k}(X)=\mathbf{c} \mu_{k}(X)$ for all $k$.

To complete the proof of Theorem 4, we use the following lemma.

LEMMA 2。If $\mu_{1}, \cdots, \mu_{p}$ are finite and atomless and $c_{1}, \cdots, c_{n}$ are nonnegative numbers satisfying $c_{1}+\cdots+c_{n}=1$, then there exists a decomposition of $X$ into $n$ dis joint measurable sets $S_{1}, \cdots, S_{n}$ having the property that

$$
\mu_{k}\left(S_{j}\right)=c_{j} \mu_{k}(X) \quad(j=1, \cdots, n ; k=1, \cdots, p) .
$$

Indeed, according to (13) there exists a measurable $S_{1}$ satisfying (14) for $j=1$. Similarly, there exists a measurable $S_{2} \subset X-S_{1}$ satisfying

$$
\mu_{k}\left(S_{2}\right)=\frac{c_{2}}{c_{2}+\cdots+c_{n}} \mu_{k}\left(X-S_{1}\right)=c_{2} \mu_{k}(X),
$$


where we interpret

$$
\frac{c_{2}}{c_{2}+\cdots+c_{n}}
$$

as zero if $c_{2}=\cdots=c_{n}=0$. That is, $S_{2}$ satisfies (14) for $j=2$. In the same manner $S_{j} \subset X-\mathrm{U}_{i=1}^{j-1} S_{i}$ satisfying (14) may be obtained for $j=1, \cdots, n-1$. But then

$$
\mu_{k}\left(X-\bigcup_{j=1}^{n^{-1}} S_{j}\right)=1-\left(c_{1}+\cdots+c_{n-1}\right) \mu_{k}(X)=c_{n} \mu_{k}(X)
$$

thus

$$
S_{n}=X-\bigcup_{j=1}^{n-1} S_{j}
$$

satisfies (14) for $j=n$ as required. Hence, Lemma 2 holds.

The proof of Theorem 4 can now easily be completed. Let $\eta^{0}(x)$ be any step $n$-vector. Then $X$ can be decomposed into a finite number of disjoint measurable subsets $Y_{t}$ over each of which all the components of $\eta^{0}(x)$ are constant. According to Lemma 2, $Y_{t}$ may be decomposed into $n$ disjoint measurable sets $S_{1, t}, \cdots, S_{n, t}$ such that

$$
\mu_{k}\left(S_{j, t}\right)=\int_{Y_{t}} \eta_{j}^{0}(x) d \mu_{k}(x) \quad(j=1, \cdots, n ; k=1, \cdots, p)
$$

Putting $S_{j}=\mathrm{U}_{t} S_{j, t}(j=1, \cdots, n)$ we have, from (15),

$$
\int_{X} \eta_{\jmath}{ }^{0}(x) d \mu_{k}(x)=\mu_{k}\left(S_{\jmath}\right) \quad(j=1, \cdots, n ; k=1, \cdots, p) .
$$

Thus the point $v\left(\eta^{0} ; \mu_{1}, \cdots, \mu_{p}\right)$ coincides with $v\left(\eta^{*} ; \mu_{1}, \cdots, \mu_{p}\right)$, where $\eta_{j}^{*}(x)=1$ if $x \in S_{j}$ and zero otherwise. In other words, $V_{n}^{0} \subset V_{n}^{*}$. Since the converse inclusion is obvious, Theorem 4 is proved.

Remarks. (a) Liapounoff [5] proved that if the conditions of Theorem 4 are satisfied then the set of all points $\left[\mu_{1}(S), \cdots, \mu_{p}(S)\right]$ in Euclidean $p$-space 
corresponding to all measurable $S$ is convex and compact. This result is clearly implied by the convexity and compactness of $V_{n}^{*}$; thus the convexity and compactness of $V_{n}^{*}$ may be considered as a generalization of Liapounoff's theorem. If we put $\bar{S}=X-S$, then Liapounoff's result is easily seen to be equivalent to the statement that the set of all points $\left[\mu_{1}(S), \cdots, \mu_{p}(S), \mu_{1}(\bar{S}), \cdots, \mu_{p}(\bar{S})\right]$ in Euclidean $2 p$ dimensional space is convex and compact. But this amounts precisely to the assertion that $V_{n}^{*}$ is convex and compact for $n=2$. That this assertion remains valid also for $n>2$ is precisely the generalization of Liapoun off's result contained in The orem 4 .

We used in our proof the convexity part of Liapounoff's result. This is, however, the easier part (cf. Halmos [3]), and thus our method furnishes also a new proof of Liapounoff's the orem.

(b) The values 0 and 1 are among those which the components of $\eta^{0}$ in Theorem 3 are allowed to assume. Hence, on combining the results of Theorems 3 and 4 we see that, if all but $p^{\prime}$ of the measures $\mu_{1}, \cdots, \mu_{p}$ are atomless, we may replace $p$ by $p^{\prime}$ in the exponent of 2 in Theorem 3. This estimate is again independent of the number of atoms.

(c) If the measures $\mu_{1}, \cdots, \mu_{p}$ in Theorem 4 are not assumed to be atomless, then of course $V_{n}^{*}$ need not be convex. It is, however, compact as can easily be seen on decomposing into atomless and purely atomic parts and dealing separately with each (see, for example, [3]).

(d) For some applications the following is of importance: If $\eta$ is a probability $n$-vector, then there exists a decomposition $n$-vector $\eta^{*}$ with $v\left(\eta^{*}\right)=v(\eta)$ having the further property that, for every $x \in X$ and $j=1, \cdots, n$, the vanishing of $\eta_{j}(x)$ implies that of $\eta_{j}^{*}(x)$. This assertion follows easily from Theorem 4. Indeed, $X$ may be decomposed into a finite number of measurable sets $Y$ with the following property: If $\eta_{j}(x)=0$ for some $x \in Y$, then $\eta_{j}(x)=0$ for all $x \in Y$. Let $j_{1}, \cdots, j_{m}$ be those $j$ for which $\eta_{j}(x)>0$ when $x \in Y$. We may now define $\eta^{*}(x)$ for $x \in Y$ by applying Theorem 4 (with $X$ replaced by $Y$ and $n$ by $m$ ) to the $m$-vector formed by these components, and putting $\eta_{j}(x)=0$ for all other $j$ and $x \in Y$. Combining these definitions for all sets $Y$, we obtain an $\eta^{*}$ with the required property.

4. Extension to arbitrary atomless measures. The assumption of finiteness in Theorem 4 is unnecessary. Indeed, we shall prove the following result.

THEOREM 5. If the measures $\mu_{1}, \cdots, \mu_{p}$ are atomless, then, for every n, 
the range $V_{n}$ and the decomposition range $V_{n}^{*}$ are identical.

Since the measures are now allowed to assume infinite values, the components of $V(\eta)$ are no longer necessarily finite and one should look upon $V_{n}$ and $V_{n}^{*}$ as imbedded in Euclidean space extended by allowing each coordinate to assume also infinite values.

Before proceeding to the proof we establish the following lemmas.

LEMMA 3. If $\mu$ is a nonnegative atomless measure with $\mu(X)=\infty$, and $u$ is any finite positive number, then there exists a measurable set $T$ with $\mu(T)=u$.

Proof. Since $\mu$ is nonnegative and atomless, there exists a set $S$ with $0<$ $\mu(S)<\infty$. We first show that $a=\sup \mu$ for all such sets $S$ is infinite. Indeed, assume $a$ finite; then, for every integer $m$, there exists a measurable $S_{m}$ with $\mu\left(S_{m}\right)>a-1 / m$. Put $S^{\prime}=\bigcup_{m=1}^{\infty} S_{m}$; then $\mu\left(S^{\prime}\right)=\alpha$. But $\mu\left(X-S^{\prime}\right)=\infty$; hence, there exists a measurable $S^{\prime \prime} \subset X-S^{\prime}$ with $0<\mu\left(S^{\prime \prime}\right)=b<\infty$. Thus $a<\mu\left(S^{\prime} \cup S^{\prime \prime}\right)$, contradicting the assumption that $\alpha$ is finite.

Therefore, given $u$ there exists a measurable $T^{\prime}$ with $u<\mu\left(T^{\prime}\right)<\infty$. But then, according to the intermediary values theorem of Sierpinski (see, for example, $[2,52])$, or the one dimensional case of Liapounoff's theorem, there exists a measurable $T \subset T^{\prime}$ with $\mu(T)=u$.

LEMMA 4. If $\mu$ is a nonnegative atomless measure with $\mu(X)=\infty$, and $q$ is any positive integer, then $X$ may be decomposed into $q$ measurable disjoint sets $X_{1}, \cdots, X_{q}$ with $\mu\left(X_{1}\right)=\cdots=\mu\left(X_{q}\right)=\infty$.

Proof. According to Lemma 3, there exist a set $T_{1}$ with $\mu\left(T_{1}\right)=1$, a set $T_{2} \subset X-T_{1}$ with $\mu\left(T_{2}\right)=1$, a set $T_{3} \subset X-\left(T_{1} \cup T_{2}\right)$ with $\mu\left(T_{3}\right)=1$, and so on. Putting $X_{i}=\cup_{n=0}^{\infty} T_{q n+i}$ for $i=1, \cdots, q-1$ and $X_{q}=X-\cup_{i=1}^{q-1} X_{i}$ we obtain the required result.

LEMMA 5. If $\nu_{1}, \cdots, \nu_{m}$ are nonnegative atomless measures with $\nu_{1}(X)$ $=\cdots \nu_{m}(X)=\infty$, and $q$ is any positive integer, then $X$ may be decomposed into $q$ measurable sets $X_{1}, \cdots, X_{q}$ satisfying $\nu_{i}\left(X_{1}\right)=\cdots=\nu_{i}\left(X_{q}\right)=\infty$ for $i=1, \cdots, m$.

Proof. For $m=1$, this lemma reduces to the preceding one. Assume $m>1$ and the lemma proved for $m-1$. According to Lemma $4, X$ is the union of $m$ disjoint 
measurable sets $Y_{1}, \cdots, Y_{m}$ with $\nu_{m}\left(Y_{1}\right)=\cdots=\nu_{m}\left(Y_{m}\right)=\infty$. For every $i(i=1, \cdots, m-1)$ let $i$ ' denote the smallest integer for which $\nu_{i}\left(Y_{i^{\prime}}\right)=\infty$. (Since $\nu_{i}(X)=\infty$ we have $1 \leq i^{\prime} \leq m$.) Put $Y^{\prime}=\cup_{i=1}^{m-1} Y_{i^{\prime}}$ and $Y^{\prime \prime}=X-Y^{\prime}$. Then $\nu_{m}\left(Y^{\prime \prime}\right)=\propto$ and $Y^{\prime \prime}$ is the union of disjoint measurable sets $Y_{1}^{\prime \prime}, \cdots, Y_{q}^{\prime \prime}$ with $\nu_{m}\left(Y_{1}^{\prime \prime}\right)=\cdots=\nu_{m}\left(Y_{q}^{\prime \prime}\right)=\infty$. Also $\nu_{i}\left(Y^{\prime \prime}\right)=\infty$ for $i=1, \cdots, m-1$ and hence, by the assumption of induction, it can be decomposed into measurable sets $Y_{1}^{\prime}, \cdots, Y_{q}^{\prime}$ with $\nu_{i}\left(Y_{1}^{\prime}\right)=\cdots=\nu_{i}\left(Y_{q}^{\prime}\right)$ for $i=1, \cdots, m-1$. Putting $X_{1}=Y_{1}^{\prime} \cup Y_{1}^{\prime \prime}, \cdots, X_{q}=Y_{q}^{\prime} \cup Y_{q}^{\prime \prime}$, we obtain the required decomposition.

Lemma 6. Let $\mu, \nu$ be nonnegative atomless measures with $\mu(X)<\infty$, $\nu(X)=\infty$. Then either $X$ may be decomposed into countably many measurable sets, each having finite $\nu$ measure, or there exists a measurable set $T$ with $\mu(T)=0, \nu(T)=\infty$.

Proof. For every positive integer $t$ consider the measure $\mu_{t}$ defined by

$$
\mu_{t}(S)=\nu(S)-t \mu(S) \text {. }
$$

According to Hahn (see for example [2,p.18] or [4,p.121]) $X$ may be decomposed into two disjoint measurable sets $Y_{t}$ and $\bar{Y}_{t}$ with $\mu_{t}(S) \leq 0$ for every measurable $S \subset Y_{t}$ and $\mu_{t}(S)>0$ for every measurable $S \subset \bar{Y}$. Clearly,

$$
\nu\left(Y_{t}\right) \leq t \mu\left(Y_{t}\right) \leq t \mu(X)<\infty .
$$

Put now $Y_{t}^{\prime}=Y_{1} \cup \cdots \cup Y_{t}$ and $Z_{1}=Y_{1}^{\prime}, Z_{t}=Y_{t}^{\prime}-Y_{t-1}^{\prime} \quad$ for $t=2,3, \cdots$, and denote by $Z_{0}$ the complement of $\cup_{t=1}^{\infty} Z_{t}$. Then $X=\cup_{t=0}^{\infty} Z_{t}$ and $\nu\left(Z_{t}\right)<\infty$ for $t \geq 1$. If $\nu\left(Z_{0}\right)<\infty$ then this is a decomposition of $X$ into countably many sets of finite $\nu$ measure. If, on the other hand, $\nu\left(Z_{0}\right)=\infty$ then, by Lemma 3, there exists for every integer $u$ a measurable $T_{u} \subset Z_{0}$ with $\nu\left(T_{u}\right)=u$. Moreover, $\mu\left(T_{u}\right)=0$ since, according to the construction of $Z_{0}, \mu(S)>0$ for $S \subset Z_{0}$ implies $\nu(S)=\infty$. Thus $T=\cup_{u=1}^{\infty} T_{u}$ has the properties required in Lemma 6 .

Proof of Theorem 5. Since every measure is the difference between two nonnegative measures, we may assume throughout the proof that the measures $\mu_{k}(k=1, \cdots, p)$ are nonnegative.

Let $\eta$ be any probability $n$-vector. For every $j(j=1, \cdots, n)$ we denote by $Y_{j, 0}$ the set of $x$ for which $\eta_{j}(x)=0$ and by $Y_{j, t}(t=1,2, \cdots)$ the set of $x$ for which

$$
\frac{1}{t+1}<\eta_{J}(x)<\frac{1}{t}
$$


We use $Y$ to denote any set of the form

$$
\bigcap_{j=1}^{n} Y_{j t_{j}} \quad \text { with } t_{j}=0,1,2, \cdots \quad \quad(j=1, \cdots, n)
$$

The space $X$ is thus decomposed into countably many sets $Y$ having the following property: There exists a nonempty subset $J=J(Y)$ of $\{1, \cdots, n\}$ and a positive $\delta=\delta(Y)$ such that for all $x \in Y$ we have

$$
\eta_{j}(x)>\delta>0 \quad \text { if } j \in J, \quad \eta_{j}(x)=0 \quad \text { if } j \notin J .
$$

Let $Y$ be any such set and consider the subset $K^{\prime}$ of $\{1, \cdots, p\}$ consisting of all those $k$ for which $Y$ can be decomposed into countably many sets, all having finite $\mu_{k}$ measure. If $K^{\prime}$ is empty, we call $Y$ final, if not we decompose $Y$ into countably many measurable sets $Y^{\prime}$ with $\mu_{k}\left(Y^{\prime}\right)<\infty$ for $k \in K^{\prime}$. Let $Y^{\prime}$ be any such set and denote by $K^{\prime \prime}$ the subset of $\{1, \cdots, p\}$ consisting of all $k$ for which $Y^{\prime}$ can be decomposed into countably many sets, all having finite $\mu_{k}$ measure. Clearly, $K^{\prime} \subset K^{\prime \prime}$. If $K^{\prime}=K^{\prime \prime}$ we call $Y^{\prime}$ final, if not we decompose it into countably many $Y^{\prime \prime}$ with $\mu_{k}\left(Y^{\prime \prime}\right)<\infty$ for $k \in K^{\prime \prime}$. Again a $K^{\prime \prime \prime} \supset K^{\prime \prime}$ is defined and $Y^{\prime \prime}$ is called final if $K^{\prime \prime}=K^{\prime \prime \prime}$, and so on. After not more than $p$ steps we always end with a final set $Z$.

We have thus decomposed $Y$, and hence $X$, into countably many sets $Z$ having the following property: To every $Z$ there corresponds a decomposition of $\{1,2$, $\cdots, p\}$ into two disjoint sets $K$ and $K$ such that $\mu_{k}(Z)<\infty$ if $k \in K$, while if $k \in \bar{K}$ then $Z$ cannot be decomposed into countably many sets, all having finite $\mu_{k}$ measure. Furthermore, since $Z$ is contained in some $Y$, (16) holds for all $x \in Z$.

Next, we show how to decompose $Z$ into disjoint measurable sets $Z_{1}, \cdots, Z_{n}$ satisfying

$$
\mu_{k}\left(Z_{j}\right)=\int_{Z} \eta_{j}(x) d \mu_{k}(x) \quad(j=1, \cdots, n ; k=1, \cdots, p) .
$$

(If $\eta_{j}(x)=0$ for all $x \in Z$, the right side of (17) is understood to be 0 even when $\mu_{k}(Z)=\infty$.)

If $K$ is empty, then the possibility of such a decomposition is assured by Theorem 4.

If $K$ is empty then, by (16), the integral in (17) is infinite if $j \in J$ and is zero 
otherwise. By Lemma 5 it is possible to decompose $Z$ into sets $Z_{j}(j \in J)$ with

$$
\mu_{k}\left(Z_{\jmath}\right)=\infty \quad \text { for } k=1, \cdots, p \text {. }
$$

Denoting the empty set, for $j \notin J$, by $Z_{j}$, we have a decomposition satisfying (17).

Finally, assume both $K$ and $\bar{K}$ nonempty. We define a nonnegative measure $\mu$ by $\mu(S)=\Sigma_{k \in K} \mu_{k}(S)$. Clearly, $\mu$ is atomless and $\mu(Z)<\infty$. According to Lemma 6 there exists, for every $k \in \bar{K}$, a measurable $T_{k} \subset Z$ with $\mu\left(T_{k}\right)=0$, $\mu_{k}\left(T_{k}\right)=\infty$. Let $T$ be the union of $T_{k}(k \in \bar{K})$. Then (see the treatment of the case when $K$ is empty) it is possible to decompose $T$ into disjoint measurable sets $Z_{1}^{\prime}, \cdots, Z_{n}^{\prime}$ so that $Z_{j}^{\prime}$ is empty for $j \notin J$, while for all $j \in J$ and $k \in \bar{K}$ we have $\mu_{k}\left(Z_{j}^{\prime}\right)=\infty$. Since $\mu(T)=0$ we have, for all $j, \mu_{k}\left(Z_{j}^{\prime}\right)=0$ whenever $k \in K$. Let $T^{\prime}=Z-T$; then it is possible, by Theorem 4 , to decompose $T^{\prime}$ into disjoint measurable sets $T_{1}^{\prime}, \cdots, T_{n}^{\prime}$ such that $T_{j}^{\prime}$ is empty for $j \notin J$, while for $j \in J$ and $k \in K$ we have

$$
\mu_{k}\left(Y_{j}\right)=\int_{T^{\prime}} \eta_{j}(x) d \mu_{k}(x)=\int_{Z} \eta_{j}(x) d \mu_{k}(x) .
$$

Putting $Z_{j}=T_{j}^{\prime} \cup Z_{j}^{\prime}$ for $j=1, \cdots, n$, we have a decomposition satisfying (17).

We now define the decomposition $n$-vector $\eta^{*}$ as follows: For $x \in Z$, put $\eta_{j}^{*}(x)=1$ if $x \in Z_{j}$, and $\eta_{j}^{*}(x)=0$ for all other $x \in Z$. Because of the countable additivity of the measures and the integrals, (17) implies $v\left(\eta^{*}\right)=v(\eta)$ and the proof is completed.

Remarks. (a) The last remark after Theorem 4 applies also here. Indeed, our construction in the proof of Theorem 5 yields a vector having the properties required of $\eta^{*}$ in that remark.

(b) In applications usually $X$ can be decomposed into countably many sets of finite $\mu_{k}$ measure $(k=1, \cdots, p)$. For this special case Theorem 5 is, of course, an immediate consequence of Theorem 4 .

4. Application to statistics and the theory of games.* Theorem 4 (together with its extension mentioned in the last remark of the preceding section) has

* A more detailed discussion and other results, including a discussion of the sequential statistical decision problem, are contained in our paper, Elimination of randomization in certain statistical decision procedures and zero-sum two-person games, Annals of Mathematical Statistics, 22, No. 1, March, 1951. A brief discussion of these applications was also given in an earlier publication $[1]$. 
immediate applications to the following statistical decision problem: Let $y=$ $\left\{y_{1}, \cdots, y_{t}\right\}$ be a random vector with $t$ components, where $t$ is a given positive integer. For every point $x=\left(x_{1}, \cdots, x_{t}\right)$ of the $t$-dimensional Euclidean space $X$, let $F(x)$ denote the probability that $y_{i}<x_{i}$ for $i=1, \cdots, t$; that is, $F(x)$ is the distribution function of $y$. The distribution function $F(x)$ is assumed to be unknown. It is known, however, that $F(x)$ is one of the distribution functions $F_{1}(x), \cdots, F_{m}(x)$. An observation $x$ is made on $y$ and according to the observed value $x$ the statistician may adopt any one of $n$ decisions $j(j=1, \cdots, n)$. Let $W_{i, j}(x)$ denote the loss sustained by the statistician when $F_{i}(x)$ is the true distribution of $y, x$ is the observed value of $y$, and the $j$ th decision is adopted. $W_{i, j}(x)$ is assumed to be a finite nonnegative and measurable function of $x$. If the statistician, on observing the value $x$, adopts the various decisions with probabilities $\eta_{j}(x)$, where these are nonnegative measurable functions satisfying (1), then the risk, or expected loss, when $F_{i}(x)$ is the true distribution function, is given by

$$
r_{\imath}(\eta)=\sum_{j=1}^{n} \int_{X} W_{\imath, j}(x) \eta_{J}(x) d F_{\imath}(x) .
$$

The decision function $\eta_{j}(x)$ is said to be nonrandomized if for every $x$ all but one of the $\eta_{j}(x)$ vanish. Theorem 4 yields without difficulty the following result: If the distribution functions $F_{i}(x)(i=1, \cdots, m)$ are atomless then, given any decision function $\eta(x)$, there exists a nonrandomized decision function $\eta^{*}(x)$ such that $r_{i}(\eta)=r_{i}\left(\eta^{*}\right)(i=1, \cdots, m)$.

Similar application can be made to the theory of games. In fact, the above described statistical decision problem may be interpreted as a zero-sum two-person game as follows: Player 1 has a finite number of pure strategies $i(i=1, \cdots, m)$, while a pure strategy of Player 2 is a nonrandomized decision function $\eta^{*}(x)$ (decomposition $n$-vector). If $i$ is the pure strategy of Player 1 and $\eta^{*}(x)$ the pure strategy of Player 2, the outcome is defined by

$$
R\left[i, \eta^{*}(x)\right]=r_{i}\left(\eta^{*}\right) .
$$

A mixed strategy of Player 1 is represented by a vector $\xi=\left(\xi_{1}, \cdots, \xi_{m}\right)$ with nonnegative components whose sum is one, while a mixed strategy of Player 2 is given by a probability $n$-vector $\eta(x)$. The expected value of the outcome corresponding to the mixed strategies $\xi$ and $\eta(x)$ is given by 


$$
R[\xi, \eta(x)]=\sum_{i=1}^{m} \xi_{i} r_{i}(\eta)
$$

The above stated result for the statistical decision problem can be restated in game terminology as follows: If the distribution functions $F_{i}(x)(i=1, \cdots, m)$ are atomless, then given any mixed strategy $\eta(x)$ of Player 2 , there exists a pure strategy $\eta^{*}(x)$ such that $R\left[\xi, \eta^{*}(x)\right]=R[\xi, \eta(x)]$ for all strategies $\xi$ of Player 1.

\section{REFERENCES}

1. A. Dvoretzky, A. Wald, and J. Wolfowitz, Elimination of randomization in certain problems of statistics and of the theory of games, Proc. Nat. Acad. Sci. U.S.A. 36 (1950), 256-259. .

2. H. Hahn and A. Rosenthal, Set functions, University of New Mexico Press, Albuquerque (1948).

3. P. R. Halmos, The range of a vector measure, Bull. Amer. Math. Soc. 54 (1948), 416-421.

4. Measure theory, Van Nostrand, New York (1950).

5. A. Liapounoff, Sur les fonctions-vecteurs complètement additives, Bull. Acad. Sci. URSS Sér. Math. [Izvestia Akad. Nauk SSSR] , 4 (1940), 465-478.

6. A. Wald, Statistical decision functions, Wiley, New York (1950).

National Bureau of Standards, Los Angeles

Columbia University 


\title{
EDITORS
}

\author{
HERBERT BUSEMANN \\ University of Southern California \\ Los Angeles 7, California
}

R. M. RoBINSON

University of California

Berkeley 4, California

E. F. BECKENBACH, Managing Editor

University of California

Los Angeles 24, California

ASSOCIATE EDITORS
R. P. DILWORTH
P. R. HALMOS
BQRGE JESSEN
J. J. STOKER
HERBERT FEDERER
HEINZ HOPF
PAUL LÉVY
E. G. STRAUS
MARSHALL HALL
R. D. JAMES
GEORGE PÓLYA
KÔSAKU YOSIDA

\section{SPONSORS}

UNIVERSITY OF BRITISH COLUMBIA

CALIFORNIA INSTITUTE OF TECHNOLOGY UNIVERSITY OF CALIFORNIA, BERKELEY

UNIVERSITY OF CALIFORNIA, DAVIS

UNIVERSITY OF CALIFORNIA, LOS ANGELES

UNIVERSITY OF CALIFORNIA, SANTA BARBARA

OREGON STATE COLLEGE

UNIVERSITY OF OREGON
UNIVERSITY OF SOUTHERN CALIFORNIA STANFORD UNIVERSITY

WASHINGTON STATE COLLEGE

UNIVERSITY OF WASHINGTON

AMERICAN MATHEMATICAL SOCIETY NATIONAL BUREAU OF STANDARDS, INSTITUTE FOR NUMERICAL ANALYSIS

\section{Vari-Type Composition by \\ Cecile Leonard \\ Ruth Stafford}

With the cooperation of

E. F. Beckenbach

E. G. Straus

Printed in the United States of America by

Edwards Brothers, Inc., Ann Arbor, Michigan

UNIVERSITY OF CALIFORNIA PRESS - BERKELEY AND LOS ANGELES COPYRIGHT 1951 BY PACIFIC JOURNAL OF MATHEMATICS 


\section{Pacific Journal of Mathematics}

\section{Vol. 1, No. $1 \quad$ November, 1951}

Ralph Palmer Agnew, Ratio tests for convergence of series............. 1

Richard Arens and James Dugundji, Topologies for function spaces....... 5

B. Arnold, Distributive lattices with a third operation defined ........... 33

R. Bing, Concerning hereditarily indecomposable continua ........... 43

David Dekker, Generalizations of hypergeodesics ............... 53

A. Dvoretzky, A. Wald and J. Wolfowitz, Relations among certain ranges of vector measures.................................... 59

Paul Erdős, F. Herzog and G. Pirani, Schlicht Taylor series whose convergence on the unit circle is uniform but not absolute .......... 75

Whilhelm Fischer, On Dedekind's function $\eta(\tau) \ldots \ldots \ldots \ldots \ldots \ldots . \ldots 3$

Werner Leutert, The heavy sphere supported by a concentrated force ...... 97

Ivan Niven and H. Zuckerman, On the definition of normal numbers ...... 103

L. Paige, Complete mappings of finite groups .................. 111

Otto Szász, On a Tauberian theorem for Abel summability ............. 117

Olga Taussky, Classes of matrices and quadratic fields ............. 127

F. Tricomi and A. Erdélyi, The asymptotic expansion of a ratio of gamma functions .................................... 133

Hassler Whitney, On totally differentiable and smooth functions ......... 143 